Research Article

\title{
Improved Results on Reachable Set Bounding for Linear Delayed Systems with Polytopic Uncertainties
}

\author{
Hao Chen ${ }^{1,2}$ \\ ${ }^{1}$ School of Mathematical Sciences, University of Electronic Science and Technology of China, Chengdu, Sichuan 611731, China \\ ${ }^{2}$ School of Mathematical Sciences, Huaibei Normal University, Huaibei, Anhui 235000, China \\ Correspondence should be addressed to Hao Chen; chh0308@126.com
}

Received 4 December 2014; Revised 21 January 2015; Accepted 21 January 2015

Academic Editor: Luca Guerrini

Copyright (C) 2015 Hao Chen. This is an open access article distributed under the Creative Commons Attribution License, which permits unrestricted use, distribution, and reproduction in any medium, provided the original work is properly cited.

\begin{abstract}
This paper focuses on bound of reachable sets for delayed linear systems with polytopic uncertainties. Based on LyapunovKrasovskii functional theory, delay decomposition technique, and reciprocally convex method, some new results expressed in the form of linear matrix inequalities are derived. It should be noted that triple integral functionals are first to be introduced for reachable set analysis. Consequently, a tighter bound of the reachable set is obtained. Four numerical examples are given to illustrate the effectiveness and advantage of the proposed results comparing with the existing criteria.
\end{abstract}

\section{Introduction}

In real world, many phenomena can be described by time delay systems, such as communication networks, biology, and physical process. It is well known that the presence of time delay may lead to complicated behaviors for dynamic system, including instability, oscillations, and robustness [1-5]. In addition to stability and robustness of the state, the property of input-to-state for dynamical systems is also concerned. For a dynamic system, reachable set is the set of all the states in the Euclidean space that are reachable from the origin, in finite time, by inputs with peak value that is bounded by some given positive scalar [6]. It was first considered in the late 1960s and it has a wide range of applications, such as peak-to-peak gain minimization problem and control systems with actuator saturation. Thus, the problem of reachable set bounding for time delay systems has received considerable attention in recent years; for instance, see [6-18] and the references therein.

There are already some relevant results about the problem of reachable set bounding for linear systems. An LMI condition for an ellipsoid that bounded the reachable set of linear systems without time delay was given by Boyd in [13]. In [6], Fridman and Shaked firstly derived LMIs criteria of an ellipsoid that bounded the reachable set of uncertain systems with time-varying delays and bounded peak input based on the Razumikhin theory. In [11], Kim proposed an improved condition by using the modified LyapunovRazumikhin functionals. Nam and Pathirana obtained a smaller reachable set bound by the delay decomposition technique [10]. The maximal Lyapunov functionals, combined with the Razumikhin method, were employed to give a nonellipsoidal description of the reachable set in [15]. More recently, the authors derived the ellipsoid bounds of reachable sets of linear uncertain linear discrete-time systems based on the idea to minimize the projection distances of the ellipsoids on each axis with different exponential convergence rates [17]. Based on property of Metzler matrix, a new approach which did not involve the Lyapunov-Krasovskii functional method was used to get the state bounding for linear timedelayed systems [18]. The delays considered in $[6-9,11,12,15$, $16,18]$ are from 0 to an upper bound. However, delays may vary in an interval for which lower bound of delays is not necessary to be 0 , such as [10]. On the other hand, the authors considered nondifferentiable time-varying delays in $[10,18]$, and differentiable time-varying delays were considered in [6$9,11,12,15,16]$. Paper [11] assumed the derivative of delay to be less than 1 . As is well known, large value of derivative of delay may yield bigger reachable set bounding. These constraints on the delays are strong and may be relaxed. 
In this paper, we study the reachable set bounding for linear delayed systems with polytopic uncertainties. Constraints for delay are relaxed. Time delays vary in an interval for which lower bound of delays is not necessarily 0 , and value of derivative of delay is not necessarily less than 1 . Inspired by the Lyapunov functionals in [2], we construct Lyapunov-Krasovskii functionals, combining with the delay decomposition technique and reciprocally convex method to derive a more accurate description of the reachable set bound. Different from the Lyapunov functionals in [2], the integral terms of Lyapunov functionals in this paper contain $e^{\alpha(s-t)}$. Moreover, to the best of our knowledge, it is first time to introduce triple integral functionals for reachable set analysis. We will show that the reachable set bound is tighter than that of $[6,8-12,14]$. Numerical examples illustrate the effectiveness and improvement of the obtained results.

Notations. The notations are used in this paper except where otherwise specified. $R^{n}$ is the $n$-dimension Euclidean space and $R^{n \times m}$ denotes the set of $n \times m$-dimension real matrices; real matrix $P>0(\geq 0)$ means that $P$ is a symmetric positive definite (positive semidefinite) matrix. Superscript " $T$ " denotes transposition of a vector or a matrix; $\star$ represents the elements below the main diagonal of a symmetric block matrix; I denotes an identity matrix; "--" in tables represents no feasible solution for linear matrix inequality.

\section{Preliminaries}

Consider the following uncertain polytopic time-delayed linear systems with disturbances:

$$
\begin{aligned}
\dot{z}(t)= & (A+\Delta A) z(t)+(D+\Delta D) z(t-\tau(t)) \\
& +(B+\Delta B) w(t), \quad z(t)=0, t \in\left[-\tau_{M}, 0\right],
\end{aligned}
$$

where $z(t) \in R^{n}$ is the state vector; $w(t) \in R^{m}$ is the disturbance. One has $A, \Delta A \in R^{n \times n}, D, \Delta D \in R^{n \times n}$, and $B, \Delta B \in R^{n \times m} . A, D, B$ are known constant matrices. $\tau(t)$ is the time-varying delay. For disturbance $w(t)$, we assume that $w^{T}(t) w(t) \leq w_{m}^{2}$, where $w_{m}$ is a constant.

The uncertainties are expressed as a linear convex-hull of known matrices $A_{i}, B_{i}$, and $D_{i}$ :

$$
\begin{gathered}
\Delta A=\sum_{i=1}^{N} \theta_{i}(t) A_{i}, \quad \Delta B=\sum_{i=1}^{N} \theta_{i}(t) B_{i}, \\
\Delta D=\sum_{i=1}^{N} \theta_{i}(t) D_{i},
\end{gathered}
$$

with $\theta_{i}(t) \in[0,1]$ and $\sum_{i=1}^{N} \theta_{i}(t)=1, \forall t>0$.

In this paper, time-varying delay $\tau(t)$ will be considered in two cases:

(a) $\tau_{m} \leq \tau(t) \leq \tau_{M}$,

(b) $\tau_{m} \leq \tau(t) \leq \tau_{M}, \dot{\tau}(t) \leq \mu$.

The following lemmas are useful in deriving the criteria.
Lemma 1 (see [19]). The following relation is known as the Leibniz rule:

$$
\begin{aligned}
\frac{d}{d t} \int_{a(t)}^{b(t)} f(t, s) d s= & \dot{b}(t) f[t, b(t)]-\dot{a}(t) f[t, a(t)] \\
& +\int_{a(t)}^{b(t)} \frac{\partial}{\partial t} f(t, s) d s .
\end{aligned}
$$

Lemma 2 (see [4]). For any constant matrix $P=P^{T}>0$ and $h_{2}>h_{1} \geq 0$ such that the following integrations are well defined, then

$$
\begin{aligned}
-\left(h_{2}-h_{1}\right) \int_{t-h_{2}}^{t-h_{1}} z^{T}(s) P z(s) d s \\
\quad \leq-\left(\int_{t-h_{2}}^{t-h_{1}} z(s) d s\right)^{T} P\left(\int_{t-h_{2}}^{t-h_{1}} z(s) d s\right) .
\end{aligned}
$$

Lemma 3 (see [5]). For any constant matrix $R>0$, scalars $h_{2}>h_{1} \geq 0$ such that the following integrations are well defined, then

$$
\begin{aligned}
- & \frac{1}{2}\left(h_{2}-h_{1}\right)^{2} \int_{-h_{2}}^{-h_{1}} \int_{t+\theta}^{t-h_{1}} z^{T}(s) R z(s) d s d \theta \\
& \leq-\left(\int_{-h_{2}}^{-h_{1}} \int_{t+\theta}^{t-h_{1}} z^{T}(s) d s d \theta\right) R\left(\int_{-h_{2}}^{-h_{1}} \int_{t+\theta}^{t-h_{1}} z(s) d s d \theta\right) .
\end{aligned}
$$

Proof. By using Lemma 2, one can obtain

$$
\begin{aligned}
& -\left(-h_{1}-\theta\right) \int_{t+\theta}^{t-h_{1}} z^{T}(s) R z(s) d s \\
& \quad \leq-\left(\int_{t+\theta}^{t-h_{1}} z(s) d s\right)^{T} R\left(\int_{t+\theta}^{t-h_{1}} z(s) d s\right) .
\end{aligned}
$$
holds:

According to Schur complement, the following inequality

$$
\left[\begin{array}{cc}
-\int_{t+\theta}^{t-h_{1}} z^{T}(s) R z(s) d s & \left(\int_{t+\theta}^{t-h_{1}} z(s) d s\right)^{T} \\
\star & \left(-h_{1}-\theta\right) R^{-1}
\end{array}\right] \leq 0 .
$$

Integrating both sides of the above inequality from $-h_{2}$ to $-h_{1}$, we have

$$
\left[\begin{array}{cc}
-\int_{-h_{2}}^{-h_{1}} \int_{t+\theta}^{t-h_{1}} z^{T}(s) R z(s) d s d \theta & \left(\int_{-h_{2}}^{-h_{1}} \int_{t+\theta}^{t-h_{1}} z(s) d s d \theta\right)^{T} \\
\star & \int_{-h_{2}}^{-h_{1}}\left(-h_{1}-\theta\right) d \theta R^{-1}
\end{array}\right]
$$

$\leq 0$.

By using Schur complement again, inequality (8) is equivalent to the inequality in Lemma 3 . This completes the proof. 
Lemma 4 (see [1]). Let $f_{1}, f_{2}, \ldots, f_{N}: R^{m} \mapsto R$ have positive values in an open subset $D$ of $R^{m}$. Then, the reciprocally convex combination of $f_{i}$ over $D$ satisfies

$$
\min _{\left\{\alpha_{i} \mid \alpha_{i}>0, \sum_{i=1}^{N} \alpha_{i}=1\right\}} \sum_{i=1}^{N} \frac{1}{\alpha_{i}} f_{i}(t)+\max _{g_{i, j}(t)} \sum_{i \neq j} g_{i, j}(t)
$$

subject to

$$
\left\{g_{i, j}(t): R^{m} \longmapsto R, g_{j, i}(t)=g_{i, j}(t),\left[\begin{array}{cc}
f_{i}(t) & g_{i, j}(t) \\
g_{i, j}(t) & f_{j}(t)
\end{array}\right] \geq 0\right\} .
$$

Lemma 5 (see [2]). For any vectors $z_{1}, z_{2}$, constant matrices $T_{i}$ ( $i=1,2,3,4)$, $S$, and scalars $\alpha>0, \beta>0$ satisfying $\alpha+\beta=1$, then following inequality holds:

$$
\begin{gathered}
-\frac{1}{\alpha} z_{1}^{T} T_{1} z_{1}-\frac{1}{\beta} z_{2}^{T} T_{2} z_{2}-\frac{\beta}{\alpha} z_{1}^{T} T_{3} z_{1}-\frac{\alpha}{\beta} z_{2}^{T} T_{4} z_{2} \\
\leq-\left[\begin{array}{l}
z_{1} \\
z_{2}
\end{array}\right]^{T}\left[\begin{array}{cc}
T_{1} & S \\
S^{T} & T_{2}
\end{array}\right]\left[\begin{array}{l}
z_{1} \\
z_{2}
\end{array}\right],
\end{gathered}
$$

subject to

$$
\left[\begin{array}{cc}
T_{1}+T_{3} & S \\
S^{T} & T_{2}+T_{4}
\end{array}\right] \geq 0 .
$$

Lemma 6 (see [13]). Let $V$ be a Lyapunov function for system (1) with $w^{T}(t) w(t) \leq w_{m}^{2}$. If

$$
\dot{V}+\alpha V-\frac{\alpha}{w_{m}^{2}} w^{T}(t) w(t) \leq 0,
$$

then $V \leq 1$.

\section{Main Results}

In order to study the reachable set bounding of uncertain system (1), firstly, we consider $\Delta A=0, \Delta D=0, \Delta B=0$ in system (1); that is,

$$
\begin{gathered}
\dot{z}(t)=A z(t)+D z(t-\tau(t))+B w(t), \\
z(t)=0, \quad t \in[-h, 0] .
\end{gathered}
$$

The reachable set bounding of system (14) with timevarying delay $\tau(t)$ for case (a) and case (b) is stated in Theorems 7 and 8 , respectively.

After that, the reachable set bounding of uncertain system (1) with time-varying delay $\tau(t)$ for case (a) and case (b) is stated in Theorems 9 and 10, respectively.

Theorem 7. If there exist matrices $P>0, Q_{1}>0, Q_{2}>0$, $R_{1}>0, R_{2}>0, K_{1}>0, K_{2}>0, K_{3}>0$, and $K_{4}>0, S, N$ with appropriate dimensions, and a scalar $\alpha>0$, such that the following inequalities hold,

$$
\left[\begin{array}{cc}
R_{2}+K_{3} & S \\
S^{T} & R_{2}+K_{4}
\end{array}\right] \geq 0
$$

$$
\Phi=\left[\begin{array}{ccccccccc}
\Phi_{11} & P D & \Phi_{13} & 0 & \Phi_{15} & 0 & 0 & \Phi_{18} & P B \\
\star & \Phi_{22} & \Phi_{23} & \Phi_{24} & 0 & \Phi_{26} & \Phi_{27} & D^{T} N^{T} & 0 \\
\star & \star & \Phi_{33} & \Phi_{34} & \Phi_{35} & \Phi_{36} & 0 & 0 & 0 \\
\star & \star & \star & \Phi_{44} & 0 & 0 & \Phi_{47} & 0 & 0 \\
\star & \star & \star & \star & \Phi_{55} & 0 & 0 & 0 & 0 \\
\star & \star & \star & \star & \star & \Phi_{66} & 0 & 0 & 0 \\
\star & \star & \star & \star & \star & \star & \Phi_{77} & 0 & 0 \\
\star & \star & \star & \star & \star & \star & \star & \Phi_{88} & N B \\
\star & \star & \star & \star & \star & \star & \star & \star & -\frac{\alpha}{w_{m}^{2}} I
\end{array}\right]
$$

$\leq 0$,

where

$\Phi_{11}=\alpha P+P A+A^{T} P+Q_{1}-2 e^{-\alpha \tau_{m}} K_{1}-e^{-\alpha \tau_{m}} R_{1}$,

$\Phi_{13}=-e^{-\alpha \tau_{m}} R_{1}, \quad \Phi_{15}=2 e^{-\alpha \tau_{m}} K_{1}, \quad \Phi_{18}=A^{T} N^{T}$,

$\Phi_{22}=-2 e^{-\alpha \tau_{M}} K_{3}-2 e^{-\alpha \tau_{M}} K_{4}-2 e^{-\alpha \tau_{M}} R_{2}+e^{-\alpha \tau_{M}}\left(S^{T}+S\right)$,

$\Phi_{23}=e^{-\alpha \tau_{M}} R_{2}-e^{-\alpha \tau_{M}} S^{T}, \quad \Phi_{24}=e^{-\alpha \tau_{M}} R_{2}-e^{-\alpha \tau_{M}} S$,

$\Phi_{26}=2 e^{-\alpha \tau_{M}} K_{4}, \quad \Phi_{27}=2 e^{-\alpha \tau_{M}} K_{3}$,

$\Phi_{33}=-e^{-\alpha \tau_{m}} Q_{1}+e^{-\alpha \tau_{m}} Q_{2}-e^{-\alpha \tau_{m}} R_{1}-2 e^{-\alpha \tau_{m}} K_{2}$ $-2 e^{-\alpha \tau_{M}} K_{3}-e^{-\alpha \tau_{M}} R_{2}$,

$\Phi_{34}=e^{-\alpha \tau_{M}} S, \quad \Phi_{35}=2 e^{-\alpha \tau_{m}} K_{2}, \quad \Phi_{36}=2 e^{-\alpha \tau_{M}} K_{3}$,

$\Phi_{44}=-e^{-\alpha \tau_{M}} Q_{2}-2 e^{-\alpha \tau_{M}} K_{4}-e^{-\alpha \tau_{M}} R_{2}, \quad \Phi_{47}=2 e^{-\alpha \tau_{M}} K_{4}$,

$\Phi_{55}=-2 e^{-\alpha \tau_{m}} K_{1}-2 e^{-\alpha \tau_{m}} K_{2}$,

$\Phi_{66}=-2 e^{-\alpha \tau_{M}} K_{3}-2 e^{-\alpha \tau_{M}} K_{4}$,

$\Phi_{77}=-2 e^{-\alpha \tau_{M}} K_{3}-2 e^{-\alpha \tau_{M}} K_{4}$,

$\Phi_{88}=\tau_{m}^{2} R_{1}+\left(\tau_{M}-\tau_{m}\right)^{2} R_{2}+\frac{1}{2} \tau_{m}^{2} K_{1}+\frac{1}{2} \tau_{m}^{2} K_{2}$

$+\frac{1}{2}\left(\tau_{M}-\tau_{m}\right)^{2} K_{3}+\frac{1}{2}\left(\tau_{M}-\tau_{m}\right)^{2} K_{4}-N-N^{T}$,

then the reachable sets of system (14) are bounded by a ball $B(0, r)=\left\{z \in R^{n} \mid\|z\| \leq r\right\}$ with

$$
r=\frac{1}{\sqrt{\lambda_{\min }(P)}}
$$


Proof. Construct the following Lyapunov-Krasovskii functional,

$$
V\left(z_{t}\right)=\sum_{i=1}^{7} V_{i}\left(z_{t}\right)
$$

where

$$
\begin{aligned}
V_{1}\left(z_{t}\right)= & z^{T}(t) P z(t), \\
V_{2}\left(z_{t}\right)= & \int_{t-\tau_{m}}^{t} e^{\alpha(s-t)} z^{T}(s) Q_{1} z(s) d s \\
& +\int_{t-\tau_{M}}^{t-\tau_{m}} e^{\alpha(s-t)} z^{T}(s) Q_{2} z(s) d s, \\
V_{3}\left(z_{t}\right)= & \tau_{m} \int_{-\tau_{m}}^{0} \int_{t+\theta}^{t} e^{\alpha(s-t)} \dot{z}^{T}(s) R_{1} \dot{z}(s) d s, \\
V_{4}\left(z_{t}\right)= & \left(\tau_{M}-\tau_{m}\right) \int_{-\tau_{M}}^{-\tau_{m}} \int_{t+\theta}^{t} e^{\alpha(s-t)} \dot{z}^{T}(s) R_{2} \dot{z}(s) d s, \\
V_{5}\left(z_{t}\right)= & \int_{-\tau_{m}}^{0} \int_{\eta}^{0} \int_{t+\theta}^{t} e^{\alpha(s-t)} \dot{z}^{T}(s) K_{1} \dot{z}(s) d s d \theta d \eta \\
& +\int_{-\tau_{m}}^{0} \int_{-\tau_{m}}^{\eta} \int_{t+\theta}^{t} e^{\alpha(s-t)} \dot{z}^{T}(s) K_{2} \dot{z}(s) d s d \theta d \eta, \\
V_{6}\left(z_{t}\right)= & \int_{-\tau_{M}}^{-\tau_{m}} \int_{\eta}^{-\tau_{m}} \int_{t+\theta}^{t} e^{\alpha(s-t)} \dot{z}^{T}(s) K_{3} \dot{z}(s) d s d \theta d \eta, \\
V_{7}\left(z_{t}\right)= & \int_{-\tau_{M}}^{-\tau_{m}} \int_{-\tau_{M}}^{\eta} \int_{t+\theta}^{t} e^{\alpha(s-t)} \dot{z}^{T}(s) K_{4} \dot{z}(s) d s d \theta d \eta .
\end{aligned}
$$

Taking the time derivative of $V\left(z_{t}\right)$ along the trajectory of system (14), we obtain

$$
\begin{aligned}
\dot{V}_{1}\left(z_{t}\right)= & 2 z^{T}(t) P \dot{z}(t)=-\alpha V_{1}\left(z_{t}\right)+2 z^{T}(t) P \dot{z}(t) \\
& +\alpha z^{T}(t) P z(t) \\
= & -\alpha V_{1}\left(z_{t}\right)+\alpha z^{T}(t) P z(t)+2 z^{T}(t) \\
& \cdot P(A z(t)+D z(t-\tau(t))+B w(t)), \\
\dot{V}_{2}\left(z_{t}\right)= & -\alpha V_{2}\left(z_{t}\right)+z^{T}(t) Q_{1} z(t) \\
& +e^{-\alpha \tau_{m}} z^{T}\left(t-\tau_{m}\right) Q_{2} z\left(t-\tau_{m}\right) \\
& -e^{-\alpha \tau_{m}} z^{T}\left(t-\tau_{m}\right) Q_{1} z\left(t-\tau_{m}\right) \\
& -e^{-\alpha \tau_{M}} z^{T}\left(t-\tau_{M}\right) Q_{2} z\left(t-\tau_{M}\right), \\
\dot{V}_{3}\left(z_{t}\right)= & -\alpha V_{3}\left(z_{t}\right)+\tau_{m}^{2} \dot{z}^{T}(t) R_{1} \dot{z}(t) \\
& -\tau_{m} \int_{t-\tau_{m}}^{t} e^{\alpha(s-t)} \dot{z}^{T}(s) R_{1} \dot{z}(s) d s \\
\leq & -\alpha V_{3}\left(z_{t}\right)+\tau_{m}^{2} \dot{z}^{T}(t) R_{1} \dot{z}(t) \\
& -e^{-\alpha \tau_{m}}\left(z(t)-z\left(t-\tau_{m}\right)\right)^{T} \\
& \cdot R_{1}\left(z(t)-z\left(t-\tau_{m}\right)\right),
\end{aligned}
$$

$$
\dot{V}_{4}\left(z_{t}\right)=-\alpha V_{4}\left(z_{t}\right)+\left(\tau_{M}-\tau_{m}\right)^{2} \dot{z}^{T}(t) R_{2} \dot{z}(t)
$$$$
-\left(\tau_{M}-\tau_{m}\right) \int_{t-\tau_{M}}^{t-\tau_{m}} e^{\alpha(s-t)} \dot{z}^{T}(s) R_{2} \dot{z}(s) d s
$$$$
\leq-\alpha V_{4}\left(z_{t}\right)+\left(\tau_{M}-\tau_{m}\right)^{2} \dot{z}^{T}(t) R_{2} \dot{z}(t)
$$$$
-e^{-\alpha \tau_{M}}\left(\tau_{M}-\tau_{m}\right) \int_{t-\tau(t)}^{t-\tau_{m}} \dot{z}^{T}(s) R_{2} \dot{z}(s) d s
$$$$
-e^{-\alpha \tau_{M}}\left(\tau_{M}-\tau_{m}\right) \int_{t-\tau_{M}}^{t-\tau(t)} \dot{z}^{T}(s) R_{2} \dot{z}(s) d s,
$$$$
\dot{V}_{5}\left(z_{t}\right)=-\alpha V_{5}\left(z_{t}\right)+\frac{1}{2} \tau_{m}^{2} \dot{z}(t) K_{1} \dot{z}(t)+\frac{1}{2} \tau_{m}^{2} \dot{z}(t) K_{2} \dot{z}(t)
$$$$
-\int_{-\tau_{m}}^{0} \int_{t+\theta}^{t} e^{\alpha(s-t)} \dot{z}^{T}(s) K_{1} \dot{z}(s) d s d \theta
$$$$
-\int_{-\tau_{m}}^{0} \int_{t-\tau_{m}}^{t+\theta} e^{\alpha(s-t)} \dot{z}^{T}(s) K_{2} \dot{z}(s) d s d \theta
$$$$
\leq-\alpha V_{5}\left(z_{t}\right)+\frac{1}{2} \tau_{m}^{2} \dot{z}(t) K_{1} \dot{z}(t)+\frac{1}{2} \tau_{m}^{2} \dot{z}(t) K_{2} \dot{z}(t)
$$$$
-2 e^{-\alpha \tau_{m}} \frac{1}{\tau_{m}^{2}} \int_{-\tau_{m}}^{0} \int_{t+\theta}^{t} \dot{z}^{T}(s) d s d \theta
$$$$
K_{1} \int_{-\tau_{m}}^{0} \int_{t+\theta}^{t} \dot{z}(s) d s d \theta
$$$$
-2 e^{-\alpha \tau_{m}} \frac{1}{\tau_{m}^{2}} \int_{-\tau_{m}}^{0} \int_{t-\tau_{m}}^{t+\theta} \dot{z}^{T}(s) d s d \theta
$$$$
\cdot K_{2} \int_{-\tau_{m}}^{0} \int_{t-\tau_{m}}^{t+\theta} \dot{z}(s) d s d \theta
$$$$
=-\alpha V_{5}\left(z_{t}\right)+\frac{1}{2} \tau_{m}^{2} \dot{z}(t) K_{1} \dot{z}(t)+\frac{1}{2} \tau_{m}^{2} \dot{z}(t) K_{2} \dot{z}(t)
$$$$
-2 e^{-\alpha \tau_{m}}\left(z^{T}(t)-\frac{1}{\tau_{m}} \int_{t-\tau_{m}}^{t} z^{T}(s) d s\right)
$$$$
\cdot K_{1}\left(z(t)-\frac{1}{\tau_{m}} \int_{t-\tau_{m}}^{t} z(s) d s\right)
$$$$
-2 e^{-\alpha \tau_{m}}\left(\frac{1}{\tau_{m}} \int_{t-\tau_{m}}^{t} z^{T}(s) d s-z^{T}\left(t-\tau_{m}\right)\right)
$$$$
\cdot K_{2}\left(\frac{1}{\tau_{m}} \int_{t-\tau_{m}}^{t} z^{T}(s) d s-z^{T}\left(t-\tau_{m}\right)\right) \text {, }
$$

$$
\begin{aligned}
\dot{V}_{6}\left(z_{t}\right)= & -\alpha V_{6}\left(z_{t}\right)+\frac{1}{2}\left(\tau_{M}-\tau_{m}\right)^{2} \dot{z}(t) K_{3} \dot{z}(t) \\
& -\int_{-\tau_{M}}^{-\tau_{m}} \int_{t+\theta}^{t-\tau_{m}} e^{\alpha(s-t)} \dot{z}^{T}(s) K_{3} \dot{z}(s) d s d \theta \\
\leq & -\alpha V_{6}\left(z_{t}\right)+\frac{1}{2}\left(\tau_{M}-\tau_{m}\right)^{2} \dot{z}(t) K_{3} \dot{z}(t) \\
& -e^{-\alpha \tau_{M}} \int_{-\tau_{M}}^{-\tau_{m}} \int_{t+\theta}^{t-\tau_{m}} \dot{z}^{T}(s) K_{3} \dot{z}(s) d s d \theta
\end{aligned}
$$




$$
\begin{aligned}
& =-\alpha V_{6}\left(z_{t}\right)+\frac{1}{2}\left(\tau_{M}-\tau_{m}\right)^{2} \dot{z}(t) K_{3} \dot{z}(t) \\
& -e^{-\alpha \tau_{M}} \int_{-\tau(t)}^{-\tau_{m}} \int_{t+\theta}^{t-\tau_{m}} \dot{z}^{T}(s) K_{3} \dot{z}(s) d s d \theta \\
& -e^{-\alpha \tau_{M}} \int_{-\tau_{M}}^{-\tau(t)} \int_{t+\theta}^{t-\tau(t)} \dot{z}^{T}(s) K_{3} \dot{z}(s) d s d \theta \\
& -e^{-\alpha \tau_{M}} \int_{-\tau_{M}}^{-\tau(t)} \int_{t-\tau(t)}^{t-\tau_{m}} \dot{z}^{T}(s) K_{3} \dot{z}(s) d s d \theta \\
& \dot{V}_{7}\left(z_{t}\right)=-\alpha V_{7}\left(z_{t}\right)+\frac{1}{2}\left(\tau_{M}-\tau_{m}\right)^{2} \dot{z}(t) K_{4} \dot{z}(t) \\
& -\int_{-\tau_{M}}^{-\tau_{m}} \int_{t-\tau_{M}}^{t+\theta} e^{\alpha(s-t)} \dot{z}^{T}(s) K_{4} \dot{z}(s) d s d \theta \\
& \leq-\alpha V_{7}\left(z_{t}\right)+\frac{1}{2}\left(\tau_{M}-\tau_{m}\right)^{2} \dot{z}(t) K_{3} \dot{z}(t) \\
& -e^{-\alpha \tau_{M}} \int_{-\tau_{M}}^{-\tau_{m}} \int_{t-\tau_{M}}^{t+\theta} \dot{z}^{T}(s) K_{4} \dot{z}(s) d s d \theta \\
& =-\alpha V_{7}\left(z_{t}\right)+\frac{1}{2}\left(\tau_{M}-\tau_{m}\right)^{2} \dot{z}(t) K_{4} \dot{z}(t) \\
& -e^{-\alpha \tau_{M}} \int_{-\tau(t)}^{-\tau_{m}} \int_{t-\tau(t)}^{t+\theta} \dot{z}^{T}(s) K_{4} \dot{z}(s) d s d \theta \\
& -e^{-\alpha \tau_{M}} \int_{-\tau(t)}^{-\tau_{m}} \int_{t-\tau_{M}}^{t-\tau(t)} \dot{z}^{T}(s) K_{4} \dot{z}(s) d s d \theta \\
& -e^{-\alpha \tau_{M}} \int_{-\tau_{M}}^{-\tau(t)} \int_{t-\tau_{M}}^{t+\theta} \dot{z}^{T}(s) K_{4} \dot{z}(s) d s d \theta .
\end{aligned}
$$

Using Lemma 2,

$$
\begin{aligned}
\dot{V}_{4}\left(z_{t}\right) \leq & -\alpha V_{4}\left(z_{t}\right)+\left(\tau_{M}-\tau_{m}\right)^{2} \dot{z}^{T}(t) R_{2} \dot{z}(t) \\
& -e^{-\alpha \tau_{M}} \frac{\tau_{M}-\tau_{m}}{\tau(t)-\tau_{m}}\left(z^{T}\left(t-\tau_{m}\right)-z^{T}(t-\tau(t))\right) \\
& \cdot R_{2}\left(z\left(t-\tau_{m}\right)-z(t-\tau(t))\right) \\
& -e^{-\alpha \tau_{M}} \frac{\tau_{M}-\tau_{m}}{\tau_{M}-\tau(t)}\left(z^{T}(t-\tau(t))-z^{T}\left(t-\tau_{M}\right)\right) \\
& \cdot R_{2}\left(z(t-\tau(t))-z\left(t-\tau_{M}\right)\right) \\
= & -\alpha V_{4}\left(z_{t}\right)+\left(\tau_{M}-\tau_{m}\right)^{2} \dot{z}^{T}(t) R_{2} \dot{z}(t) \\
& -e^{-\alpha \tau_{M}} \frac{1}{\beta_{1}}\left(z^{T}\left(t-\tau_{m}\right)-z^{T}(t-\tau(t))\right) \\
& \cdot R_{2}\left(z\left(t-\tau_{m}\right)-z(t-\tau(t))\right) \\
& -e^{-\alpha \tau_{M}} \frac{1}{\beta_{2}}\left(z^{T}(t-\tau(t))-z^{T}\left(t-\tau_{M}\right)\right) \\
& \cdot R_{2}\left(z(t-\tau(t))-z\left(t-\tau_{M}\right)\right),
\end{aligned}
$$

where $\beta_{1}=\left(\tau(t)-\tau_{m}\right) /\left(\tau_{M}-\tau_{m}\right), \beta_{2}=\left(\tau_{M}-\tau(t)\right) /\left(\tau_{M}-\tau_{m}\right)$, and $\beta_{1}+\beta_{2}=1$.

Using Lemma 3,

$$
\begin{aligned}
& \dot{V}_{6}\left(z_{t}\right) \leq-\alpha V_{6}\left(z_{t}\right)+\frac{1}{2}\left(\tau_{M}-\tau_{m}\right)^{2} \dot{z}(t) K_{3} \dot{z}(t) \\
& -2 e^{-\alpha \tau_{M}} \frac{1}{\left(\tau(t)-\tau_{m}\right)^{2}}\left(\int_{-\tau(t)}^{-\tau_{m}} \int_{t+\theta}^{t-\tau_{m}} \dot{z}^{T}(s) d s d \theta\right) \\
& \cdot K_{3}\left(\int_{-\tau(t)}^{-\tau_{m}} \int_{t+\theta}^{t-\tau_{m}} \dot{z}(s) d s d \theta\right) \\
& -2 e^{-\alpha \tau_{M}} \frac{1}{\left(\tau_{M}-\tau(t)\right)^{2}}\left(\int_{-\tau_{M}}^{-\tau(t)} \int_{t+\theta}^{t-\tau(t)} \dot{z}^{T}(s) d s d \theta\right) \\
& \cdot K_{3}\left(\int_{-\tau_{M}}^{-\tau(t)} \int_{t+\theta}^{t-\tau(t)} \dot{z}(s) d s d \theta\right) \\
& -e^{-\alpha \tau_{M}} \frac{\tau_{M}-\tau(t)}{\tau(t)-\tau_{m}}\left(\int_{t-\tau(t)}^{t-\tau_{m}} \dot{z}^{T}(s) d s d \theta\right) \\
& K_{3}\left(\int_{t-\tau(t)}^{t-\tau_{m}} \dot{z}^{T}(s) d s d \theta\right) \\
& =-\alpha V_{6}\left(z_{t}\right)+\frac{1}{2}\left(\tau_{M}-\tau_{m}\right)^{2} \dot{z}(t) K_{3} \dot{z}(t) \\
& -2 e^{-\alpha \tau_{M}}\left(z^{T}\left(t-\tau_{m}\right)-\frac{1}{\tau(t)-\tau_{m}} \int_{t-\tau(t)}^{t-\tau_{m}} z^{T}(s) d s\right) \\
& \cdot K_{3}\left(z\left(t-\tau_{m}\right)-\frac{1}{\tau(t)-\tau_{m}} \int_{t-\tau(t)}^{t-\tau_{m}} z(s) d s\right) \\
& -2 e^{-\alpha \tau_{M}}\left(z(t-\tau(t))-\frac{1}{\tau_{M}-\tau(t)} \int_{t-\tau_{M}}^{t-\tau(t)} z(s) d s\right)^{T} \\
& \cdot K_{3}\left(z(t-\tau(t))-\frac{1}{\tau_{M}-\tau(t)} \int_{t-\tau_{M}}^{t-\tau(t)} z(s) d s\right) \\
& -e^{-\alpha \tau_{M}} \frac{\beta_{2}}{\beta_{1}}\left(z^{T}\left(t-\tau_{m}\right)-z^{T}(t-\tau(t))\right) \\
& \cdot K_{3}\left(z\left(t-\tau_{m}\right)-z(t-\tau(t))\right), \\
& \dot{V}_{7}\left(z_{t}\right) \leq-\alpha V_{7}\left(z_{t}\right)+\frac{1}{2}\left(\tau_{M}-\tau_{m}\right)^{2} \dot{z}(t) K_{4} \dot{z}(t) \\
& -2 e^{-\alpha \tau_{M}} \frac{1}{\left(\tau(t)-\tau_{m}\right)^{2}}\left(\int_{-\tau(t)}^{-\tau_{m}} \int_{t-\tau(t)}^{t+\theta} \dot{z}^{T}(s) d s d \theta\right) \\
& \cdot K_{4}\left(\int_{-\tau(t)}^{-\tau_{m}} \int_{t-\tau(t)}^{t+\theta} \dot{z}^{T}(s) d s d \theta\right) \\
& -e^{-\alpha \tau_{M}} \frac{\tau(t)-\tau_{m}}{\tau_{M}-\tau(t)}\left(\int_{t-\tau_{M}}^{t-\tau(t)} \dot{z}^{T}(s) d s d \theta\right) \\
& \cdot K_{4}\left(\int_{t-\tau_{M}}^{t-\tau(t)} \dot{z}(s) d s d \theta\right)
\end{aligned}
$$




$$
\begin{aligned}
& -2 e^{-\alpha \tau_{M}} \frac{1}{\left(\tau_{M}-\tau(t)\right)^{2}}\left(\int_{-\tau_{M}}^{-\tau(t)} \int_{t-\tau_{M}}^{t+\theta} \dot{z}^{T}(s) d s d \theta\right) \\
& \cdot K_{4}\left(\int_{-\tau_{M}}^{-\tau(t)} \int_{t-\tau_{M}}^{t+\theta} \dot{z}(s) d s d \theta\right) \\
= & -\alpha V_{7}\left(z_{t}\right)+\frac{1}{2}\left(\tau_{M}-\tau_{m}\right)^{2} \dot{z}(t) K_{4} \dot{z}(t) \\
& -2 e^{-\alpha \tau_{M}}\left(\frac{1}{\tau(t)-\tau_{m}} \int_{t-\tau(t)}^{t-\tau_{m}} z^{T}(s) d s-z(t-\tau(t))\right) \\
& \cdot K_{4}\left(\frac{1}{\tau(t)-\tau_{m}} \int_{t-\tau(t)}^{t-\tau_{m}} z(s) d s-z(t-\tau(t))\right) \\
& -e^{-\alpha \tau_{M}} \frac{\beta_{1}}{\beta_{2}}\left(z^{T}(t-\tau(t))-z^{T}\left(t-\tau_{M}\right)\right) \\
& \cdot K_{4}\left(z(t-\tau(t))-z\left(t-\tau_{M}\right)\right) \\
& -2 e^{-\alpha \tau_{M}\left(\frac{1}{\tau_{M}-\tau(t)} \int_{t-\tau_{M}}^{t-\tau(t)} z^{T}(s) d s-z^{T}\left(t-\tau_{M}\right)\right)} \\
\cdot & K_{4}\left(\frac{1}{\tau_{M}-\tau(t)} \int_{t-\tau_{M}}^{t-\tau(t)} z(s) d s-z\left(t-\tau_{M}\right)\right) .
\end{aligned}
$$

From Lemma 5 and inequality (15), one can obtain

$$
\begin{aligned}
& -e^{-\alpha \tau_{M}} \frac{1}{\beta_{1}}\left(z^{T}\left(t-\tau_{m}\right)-z^{T}(t-\tau(t))\right) \\
& \cdot R_{2}\left(z\left(t-\tau_{m}\right)-z(t-\tau(t))\right) \\
& -e^{-\alpha \tau_{M}} \frac{1}{\beta_{2}}\left(z^{T}(t-\tau(t))-z^{T}\left(t-\tau_{M}\right)\right) \\
& \cdot R_{2}\left(z(t-\tau(t))-z\left(t-\tau_{M}\right)\right) \\
& -e^{-\alpha \tau_{M}} \frac{\beta_{2}}{\beta_{1}}\left(z^{T}\left(t-\tau_{m}\right)-z^{T}(t-\tau(t))\right) \\
& \cdot K_{3}\left(z\left(t-\tau_{m}\right)-z(t-\tau(t))\right) \\
& -e^{-\alpha \tau_{M}} \frac{\beta_{1}}{\beta_{2}}\left(z^{T}(t-\tau(t))-z^{T}\left(t-\tau_{M}\right)\right) \\
& \cdot K_{4}\left(z(t-\tau(t))-z\left(t-\tau_{M}\right)\right) \\
& \leq-e^{-\alpha \tau_{M}}\left[\begin{array}{ll}
z\left(t-\tau_{m}\right)-z(t-\tau(t)) \\
z(t-\tau(t))-z\left(t-\tau_{M}\right)
\end{array}\right]^{T}\left[\begin{array}{ll}
R_{2} & S \\
S^{T} & R_{2}
\end{array}\right] \\
& \cdot\left[\begin{array}{l}
z(t-\tau)-z(t-\tau(t)) \\
z(t-\tau(t))-z\left(t-\tau_{M}\right)
\end{array}\right] . \\
&
\end{aligned}
$$

Obviously, the following equation holds:

$$
2 \dot{z}^{T}(t) N(-\dot{z}(t)+A z(t)+D z(t-\tau(t))+B w(t))=0 .
$$

Combining (20)-(24), one gets

$$
\dot{V}\left(z_{t}\right)+\alpha V\left(z_{t}\right)-\frac{\alpha}{w_{m}^{2}} w^{T}(t) w(t) \leq \xi^{T}(t) \Phi \xi(t),
$$

where

$$
\begin{aligned}
\xi^{T}(t)=[ & z^{T}(t), z^{T}(t-\tau(t)), z^{T}\left(t-\tau_{m}\right), \\
& z^{T}\left(t-\tau_{M}\right), \frac{1}{\tau_{m}} \int_{t-\tau_{m}}^{t} z^{T}(s) d s, \\
& \frac{1}{\tau(t)-\tau_{m}} \int_{t-\tau(t)}^{t-\tau_{m}} z^{T}(s) d s, \\
& \left.\frac{1}{\tau_{M}-\tau(t)} \int_{t-\tau_{M}}^{t-\tau(t)} z^{T}(s) d s, \dot{z}^{T}(t), w^{T}(t)\right] .
\end{aligned}
$$

Since (15) hold, we can conclude that $\dot{V}+\alpha V-(\alpha)$ $\left.\left.w_{m}^{2}\right) w^{T}(t) w\right)(t) \leq 0$.

Therefore, one can obtain $V\left(z_{t}\right) \leq 1$ by Lemma 6 .

Using the spectral properties of symmetric positive definite matrix $P$, the following inequality holds:

$$
\lambda_{\min }(P)\|z(t)\|^{2} \leq V\left(z_{t}\right) \text {. }
$$

This further implies that $\|z(t)\| \leq r=1 / \sqrt{\lambda_{\min }(P)}$ due to (27). This completes the proof.

If the derivative of time delay $\mu$ is known, that is, the case $\tau_{m} \leq \tau(t) \leq \tau_{M}, \dot{\tau}(t) \leq \mu$, bounding for reachable set of system (14) is described in the following.

Theorem 8. If there exist matrices $P>0, Q_{1}>0, Q_{2}>0$, $R_{1}>0, R_{2}>0, K_{1}>0, K_{2}>0, K_{3}>0, K_{4}>0$, and $M>0$, $S, N$ with appropriate dimensions, and a scalar $\alpha>0$, such that the following inequalities hold,

$$
\left[\begin{array}{cc}
R_{2}+K_{3} & S \\
S^{T} & R_{2}+K_{4}
\end{array}\right] \geq 0
$$

$\Phi$

$$
\left[\begin{array}{ccccccccc}
\Phi_{11} & P D & \Phi_{13} & 0 & \Phi_{15} & 0 & 0 & \Phi_{18} & P B \\
\star & \Phi_{22} & \Phi_{23} & \Phi_{24} & 0 & \Phi_{26} & \Phi_{27} & D^{T} N^{T} & 0 \\
\star & \star & \Phi_{33} & \Phi_{34} & \Phi_{35} & \Phi_{36} & 0 & 0 & 0 \\
\star & \star & \star & \Phi_{44} & 0 & 0 & \Phi_{47} & 0 & 0 \\
\star & \star & \star & \star & \Phi_{55} & 0 & 0 & 0 & 0 \\
\star & \star & \star & \star & \star & \Phi_{66} & 0 & 0 & 0 \\
\star & \star & \star & \star & \star & \star & \Phi_{77} & 0 & 0 \\
\star & \star & \star & \star & \star & \star & \star & \Phi_{88} & N B \\
\star & \star & \star & \star & \star & \star & \star & \star & -\frac{\alpha}{w_{m}^{2}} I
\end{array}\right]
$$

$\leq 0$ 
where

$$
\begin{aligned}
& \Phi_{11}=\alpha P+P A+A^{T} P+Q_{1}-2 e^{-\alpha \tau_{m}} K_{1}^{T}-e^{-\alpha \tau_{m}} R_{1}+M, \\
& \Phi_{13}=-e^{-\alpha \tau_{m}} R_{1}, \quad \Phi_{18} \Phi_{15}=2 e^{-\alpha \tau_{m}} K_{1}, \quad \Phi_{18}=A^{T} N^{T}, \\
& \Phi_{22}=-2 e^{-\alpha \tau_{M}} K_{3}-2 e^{-\alpha \tau_{M}} K_{4}-2 e^{-\alpha \tau_{M}} R_{2} \\
& +e^{-\alpha \tau_{M}}\left(S^{T}+S\right)-(1-\mu) e^{-\alpha \tau_{M}} M, \\
& \Phi_{23}=e^{-\alpha \tau_{M}} R_{2}-e^{-\alpha \tau_{M}} S^{T} \text {, } \\
& \Phi_{24}=e^{-\alpha \tau_{M}} R_{2}-e^{-\alpha \tau_{M}} S \text {, } \\
& \Phi_{26}=-2 e^{-\alpha \tau_{M}} K_{4}, \quad \Phi_{27}=-2 e^{-\alpha \tau_{M}} K_{3}, \\
& \Phi_{33}=-e^{-\alpha \tau_{m}} Q_{1}+e^{-\alpha \tau_{m}} Q_{2}-e^{-\alpha \tau_{m}} R_{1} \\
& -2 e^{-\alpha \tau_{m}} K_{2}-2 e^{-\alpha \tau_{M}} K_{3}-e^{-\alpha \tau_{M}} R_{2} \text {, } \\
& \Phi_{34}=e^{-\alpha \tau_{M}} S, \quad \Phi_{18} \Phi_{35}=2 e^{-\alpha \tau_{m}} K_{2}, \quad \Phi_{36}=2 e^{-\alpha \tau_{M}} K_{3}, \\
& \Phi_{44}=-e^{-\alpha \tau_{M}} Q_{2}-2 e^{-\alpha \tau_{M}} K_{4}-e^{-\alpha \tau_{M}} R_{2}, \\
& \Phi_{47}=2 e^{-\alpha \tau_{M}} K_{4}, \\
& \Phi_{55}=-2 e^{-\alpha \tau_{m}} K_{1}-2 e^{-\alpha \tau_{m}} K_{2}, \\
& \Phi_{66}=-2 e^{-\alpha \tau_{M}} K_{3}-2 e^{-\alpha \tau_{M}} K_{4} \text {, } \\
& \Phi_{77}=-2 e^{-\alpha \tau_{M}} K_{3}-2 e^{-\alpha \tau_{M}} K_{4}, \\
& \Phi_{88}=\tau_{m}^{2} R_{1}+\left(\tau_{M}-\tau_{m}\right)^{2} R_{2}+\frac{1}{2} \tau_{m}^{2} K_{1}+\frac{1}{2} \tau_{m}^{2} K_{2} \\
& +\frac{1}{2}\left(\tau_{M}-\tau_{m}\right)^{2} K_{3}+\frac{1}{2}\left(\tau_{M}-\tau_{m}\right)^{2} K_{4}-N-N^{T},
\end{aligned}
$$

then the reachable sets of system (14) are bounded by a ball $B(0, r)=\left\{z \in R^{n} \mid\|z\| \leq r\right\}$ with

$$
r=\frac{1}{\sqrt{\lambda_{\min }(P)}}
$$

Proof. We modify the Lyapunov-Krasovskii functional

$$
V^{\prime}\left(z_{t}\right)=\sum_{i=1}^{7} V_{i}\left(z_{t}\right)+V_{8}\left(z_{t}\right)
$$

where

$$
V_{8}\left(z_{t}\right)=\int_{t-\tau(t)}^{t} e^{\alpha(s-t)} z^{T}(s) M z(s) d s .
$$

Taking the time derivative of $V_{8}\left(z_{t}\right)$ along the trajectory of system (14),

$$
\begin{aligned}
\dot{V}_{8}\left(z_{t}\right) \leq & -\alpha V_{8}\left(z_{t}\right)+z^{T}(t) M z(t) \\
& -(1-\mu) e^{-\alpha \tau_{M}} z^{T}(t-\tau(t)) M z(t-\tau(t)) .
\end{aligned}
$$

By the same way as in proof Theorem 7 , one can obtain the result easily. The proof is completed.

Next, consider the linear system with polytopic uncertainties (1). Bounding for reachable set of system (1) with the case $\tau_{m} \leq \tau(t) \leq \tau_{M}$ is obtained in Theorem 9 .

Theorem 9. If there exist matrices $P>0, Q_{1}>0, Q_{2}>0$, $R_{1}>0, R_{2}>0, K_{1}>0, K_{2}>0, K_{3}>0$, and $K_{4}>0, S, N$ with appropriate dimensions, and a scalar $\alpha>0$, satisfying the following inequalities for all $i=1,2, \ldots, N$,

$$
\begin{aligned}
& {\left[\begin{array}{cc}
R_{2}+K_{3} & S \\
S^{T} & R_{2}+K_{4}
\end{array}\right] \geq 0,}
\end{aligned}
$$

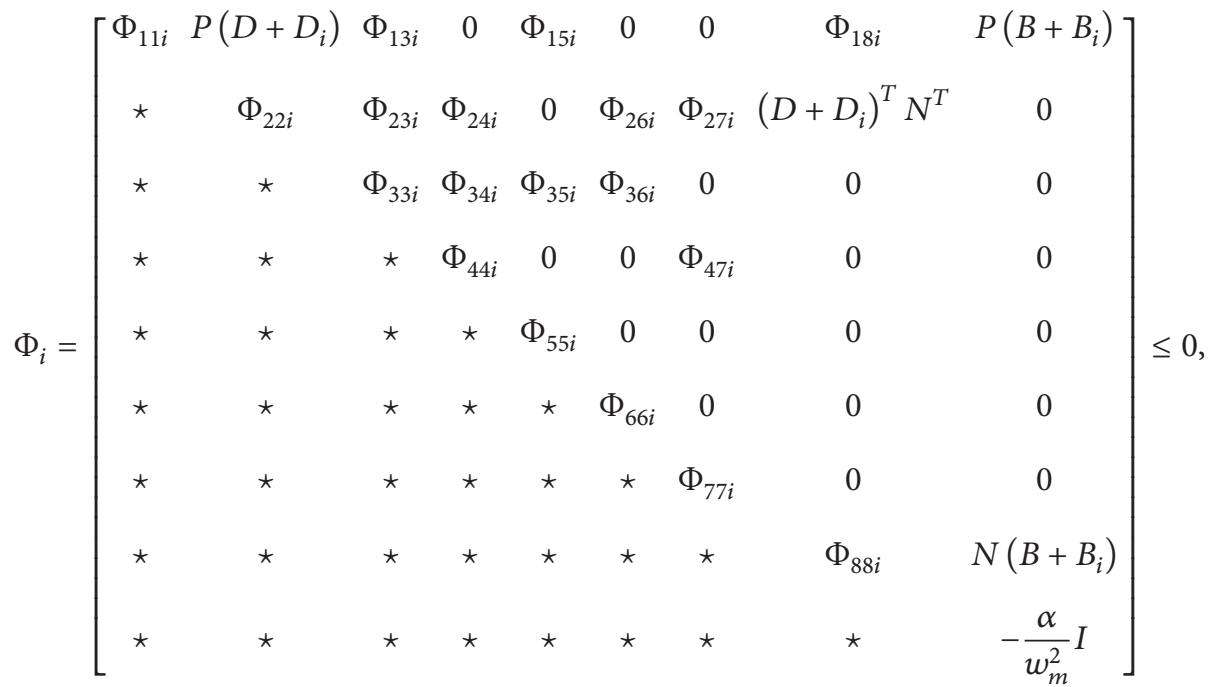


where

$$
\begin{aligned}
& \Phi_{11 i}=\alpha P+P\left(A+A_{i}\right)+\left(A+A_{i}\right)^{T} P+Q_{1} \\
& -2 e^{-\alpha \tau_{m}} K_{1}-e^{-\alpha \tau_{m}} R_{1}, \\
& \Phi_{13 i}=-e^{-\alpha \tau_{m}} R_{1}, \quad \Phi_{15}=2 e^{-\alpha \tau_{m}} K_{1}, \\
& \Phi_{18 i}=\left(A+A_{i}\right)^{T} N^{T}, \\
& \Phi_{22 i}=-2 e^{-\alpha \tau_{M}} K_{3}-2 e^{-\alpha \tau_{M}} K_{4}-2 e^{-\alpha \tau_{M}} R_{2}+e^{-\alpha \tau_{M}}\left(S^{T}+S\right), \\
& \Phi_{23 i}=e^{-\alpha \tau_{M}} R_{2}-e^{-\alpha \tau_{M}} S^{T}, \quad \Phi_{24 i}=e^{-\alpha \tau_{M}} R_{2}-e^{-\alpha \tau_{M}} S, \\
& \Phi_{26 i}=-2 e^{-\alpha \tau_{M}} K_{4}, \quad \Phi_{27 i}=-2 e^{-\alpha \tau_{M}} K_{3}, \\
& \Phi_{33 i}=-e^{-\alpha \tau_{m}} Q_{1}+e^{-\alpha \tau_{m}} Q_{2}-e^{-\alpha \tau_{m}} R_{1} \\
& -2 e^{-\alpha \tau_{m}} K_{2}-2 e^{-\alpha \tau_{M}} K_{3}-e^{-\alpha \tau_{M}} R_{2}, \\
& \Phi_{34 i}=e^{-\alpha \tau_{M}} S, \quad \Phi_{35 i}=2 e^{-\alpha \tau_{m}} K_{2}, \\
& \Phi_{36 i}=2 e^{-\alpha \tau_{M}} K_{3}, \\
& \Phi_{44 i}=-e^{-\alpha \tau_{M}} Q_{2}-2 e^{-\alpha \tau_{M}} K_{4}-e^{-\alpha \tau_{M}} R_{2}, \\
& \Phi_{47 i}=2 e^{-\alpha \tau_{M}} K_{4}, \\
& \Phi_{55 i}=-2 e^{-\alpha \tau_{m}} K_{1}-2 e^{-\alpha \tau_{m}} K_{2}, \\
& \Phi_{66 i}=-2 e^{-\alpha \tau_{M}} K_{3}-2 e^{-\alpha \tau_{M}} K_{4},
\end{aligned}
$$

TABLE 1: Computed $\bar{\delta}$ 's of Example 1 for the case $\tau_{m} \leq \tau(t) \leq \tau_{M}$ with $\tau_{m}=0$.

\begin{tabular}{lrr}
\hline Method & $\tau_{M}=0.7$ & $\tau_{M}=0.75$ \\
\hline$[6]$ & 19.71 & 65.42 \\
{$[8]$} & 5.38 & 13.44 \\
Theorem 9 & 3.91 & 6.58 \\
\hline & \\
$\Phi_{77 i}=-2 e^{-\alpha \tau_{M}} K_{3}-2 e^{-\alpha \tau_{M}} K_{4}$, & \\
$\Phi_{88 i}=\tau_{m}^{2} R_{1}+\left(\tau_{M}-\tau_{m}\right)^{2} R_{2}+\frac{1}{2} \tau_{m}^{2} K_{1}+\frac{1}{2} \tau_{m}^{2} K_{2}$ & \\
& $+\frac{1}{2}\left(\tau_{M}-\tau_{m}\right)^{2} K_{3}+\frac{1}{2}\left(\tau_{M}-\tau_{m}\right)^{2} K_{4}-N-N^{T}$,
\end{tabular}

then the reachable sets of system (1) are bounded by a ball $B(0, r)=\left\{z \in R^{n} \mid\|z\| \leq r\right\}$ with

$$
r=\frac{1}{\sqrt{\lambda_{\min }(P)}} .
$$

Proof. Replacing $A, B, D$ with $\sum_{i=1}^{N} \theta_{i}(t)\left(A+A_{i}\right), \sum_{i=1}^{N} \theta_{i}(t)(B+$ $\left.B_{i}\right)$, and $\sum_{i=1}^{N} \theta_{i}(t)\left(D+D_{i}\right)$ in proof of Theorem 7 , respectively, we easily get the conclusion.

If the derivative of time delay $\mu$ is known, that is, the case $\tau_{m} \leq \tau(t) \leq \tau_{M}, \dot{\tau}(t) \leq \mu$, bounding for reachable set of uncertain system (1) is obtained in Theorem 10.

Theorem 10. If there exist matrices $P>0, Q_{1}>0, Q_{2}>0$, $R_{1}>0, R_{2}>0, K_{1}>0, K_{2}>0, K_{3}>0, K_{4}>0$, and $M>0, S$, $N$ with appropriate dimensions, and a scalar $\alpha>0$, satisfying the following inequalities for all $i=1,2, \ldots, N$,

$$
\begin{aligned}
& {\left[\begin{array}{cc}
R_{2}+K_{3} & S \\
S^{T} & R_{2}+K_{4}
\end{array}\right] \geq 0,} \\
& \Phi_{i}=\left[\begin{array}{ccccccccc}
\Phi_{11 i} & P\left(D+D_{i}\right) & \Phi_{13 i} & 0 & \Phi_{15 i} & 0 & 0 & \Phi_{18 i} & P\left(B+B_{i}\right) \\
\star & \Phi_{22 i} & \Phi_{23 i} & \Phi_{24 i} & 0 & \Phi_{26 i} & \Phi_{27 i} & \left(D+D_{i}\right)^{T} N^{T} & 0 \\
\star & \star & \Phi_{33 i} & \Phi_{34 i} & \Phi_{35 i} & \Phi_{36 i} & 0 & 0 & 0 \\
\star & \star & \star & \Phi_{44 i} & 0 & 0 & \Phi_{47 i} & 0 & 0 \\
\star & \star & \star & \star & \Phi_{55 i} & 0 & 0 & 0 & 0 \\
\star & \star & \star & \star & \star & \Phi_{66 i} & 0 & 0 & 0 \\
\star & \star & \star & \star & \star & \star & \Phi_{77 i} & 0 & 0 \\
\star & \star & \star & \star & \star & \star & \star & \Phi_{88 i} & N\left(B+B_{i}\right) \\
\star & \star & \star & \star & \star & \star & -\frac{\alpha}{w_{m}^{2}} I
\end{array}\right] \leq 0,
\end{aligned}
$$


TABLE 2: Computed $\bar{\delta}$ 's of Example 1 for the case $\tau_{m} \leq \tau(t) \leq \tau_{M}, \dot{\tau}(t) \leq \mu$ with $\tau_{m}=0, \tau_{M}=0.7$.

\begin{tabular}{lccccccccc}
\hline$\mu$ & 0 & 0.1 & 0.2 & 0.3 & 0.4 & 0.5 & 0.6 & 0.9 & 2 \\
\hline$[11]$ & 2.97 & 3.30 & 3.85 & 4.85 & 6.93 & 12.84 & 53.86 & - & - \\
{$[8]$} & 1.89 & 1.94 & 2.00 & 2.08 & 2.19 & 2.35 & 2.60 & 3.51 & 5.30 \\
Theorem 10 & 1.79 & 1.84 & 1.90 & 1.95 & 1.96 & 1.98 & 2.06 & 3.09 & 3.23 \\
\hline
\end{tabular}

TABLE 3: Computed $\bar{\delta}$ 's of Example 1 for the case $\tau_{m} \leq \tau(t) \leq \tau_{M}, \dot{\tau}(t) \leq \mu$ with $\tau_{m}=0, \tau_{M}=0.75$.

\begin{tabular}{lccccccccc}
\hline$\mu$ & 0 & 0.1 & 0.2 & 0.3 & 0.4 & 0.5 & 0.6 & 0.9 & 2 \\
\hline$[11]$ & 3.34 & 3.79 & 4.53 & 5.88 & 8.85 & 18.36 & 127.70 & - & - \\
{$[8]$} & 2.28 & 2.35 & 2.45 & 2.57 & 2.68 & 2.85 & 4.62 & 5.57 & 13.39 \\
Theorem 10 & 2.03 & 2.33 & 2.34 & 2.45 & 2.60 & 2.72 & 4.37 & 4.92 & 8.73 \\
\hline
\end{tabular}

where

$$
\begin{aligned}
& \Phi_{11}=\alpha P+P\left(A+A_{i}\right)+\left(A+A_{i}\right)^{T} P+Q_{1}-2 e^{-\alpha \tau_{m}} K_{1} \\
& -e^{-\alpha \tau_{m}} R_{1}+M \\
& \Phi_{13}=-e^{-\alpha \tau_{m}} R_{1}, \quad \Phi_{15}=2 e^{-\alpha \tau_{m}} K_{1}, \\
& \Phi_{18}=\left(A+A_{i}\right)^{T} N^{T} \text {, } \\
& \Phi_{22}=-2 e^{-\alpha \tau_{M}} K_{3}-2 e^{-\alpha \tau_{M}} K_{4}-2 e^{-\alpha \tau_{M}} R_{2} \\
& +e^{-\alpha \tau_{M}}\left(S^{T}+S\right)-(1-\mu) e^{-\alpha \tau_{M}} M, \\
& \Phi_{23}=e^{-\alpha \tau_{M}} R_{2}-e^{-\alpha \tau_{M}} S^{T}, \quad \Phi_{24}=e^{-\alpha \tau_{M}} R_{2}-e^{-\alpha \tau_{M}} S \text {, } \\
& \Phi_{26}=-2 e^{-\alpha \tau_{M}} K_{4}, \quad \Phi_{27}=-2 e^{-\alpha \tau_{M}} K_{3}, \\
& \Phi_{33}=-e^{-\alpha \tau_{m}} Q_{1}+e^{-\alpha \tau_{m}} Q_{2}-e^{-\alpha \tau_{m}} R_{1}-2 e^{-\alpha \tau_{m}} K_{2} \\
& -2 e^{-\alpha \tau_{M}} K_{3}-e^{-\alpha \tau_{M}} R_{2}, \\
& \Phi_{34}=e^{-\alpha \tau_{M}} S, \quad \Phi_{35}=2 e^{-\alpha \tau_{m}} K_{2}, \quad \Phi_{36}=2 e^{-\alpha \tau_{M}} K_{3}, \\
& \Phi_{44}=-e^{-\alpha \tau_{M}} Q_{2}-2 e^{-\alpha \tau_{M}} K_{4}-e^{-\alpha \tau_{M}} R_{2}, \\
& \Phi_{47}=2 e^{-\alpha \tau_{M}} K_{4}, \\
& \Phi_{55}=-2 e^{-\alpha \tau_{m}} K_{1}-2 e^{-\alpha \tau_{m}} K_{2}, \\
& \Phi_{66}=-2 e^{-\alpha \tau_{M}} K_{3}-2 e^{-\alpha \tau_{M}} K_{4} \text {, } \\
& \Phi_{77}=-2 e^{-\alpha \tau_{M}} K_{3}-2 e^{-\alpha \tau_{M}} K_{4}, \\
& \Phi_{88}=\tau_{m}^{2} R_{1}+\left(\tau_{M}-\tau_{m}\right)^{2} R_{2}+\frac{1}{2} \tau_{m}^{2} K_{1}+\frac{1}{2} \tau_{m}^{2} K_{2} \\
& +\frac{1}{2}\left(\tau_{M}-\tau_{m}\right)^{2} K_{3}+\frac{1}{2}\left(\tau_{M}-\tau_{m}\right)^{2} K_{4}-N-N^{T},
\end{aligned}
$$

then the reachable sets of system (1) are bounded by a ball $B(0, r)=\left\{z \in R^{n} \mid\|z\| \leq r\right\}$ with

$$
r=\frac{1}{\sqrt{\lambda_{\min }(P)}} .
$$

Proof. Replacing $A, B, D$ with $\sum_{i=1}^{N} \theta_{i}(t)\left(A+A_{i}\right), \sum_{i=1}^{N} \theta_{i}(t)(B+$ $\left.B_{i}\right)$, and $\sum_{i=1}^{N} \theta_{i}(t)\left(D+D_{i}\right)$ in Theorem 8 , respectively, one can easily obtain the conclusion.

Remark 11. In $[7,8,12]$, the constraint of $\tau(t)$ was $0 \leq \tau(t) \leq \tau$. $\tau_{m} \leq \tau(t) \leq \tau_{M}$ is considered in this paper; it is more general than $[7,8,12]$.

Remark 12. In this paper, delay decomposition technique and reciprocally convex method are used to construct Lyapunov functionals, and triple integral terms are introduced in Lyapunov functionals for the first time to investigate bounds of reachable set for systems with uncertainties, which may lead to tighter bounding for reachable set.

Remark 13. In order to guarantee negative definite, $\mu$ is required that $\mu<1$ in [11]. It should be noted that the value of derivative of time delay is not necessarily less than 1 in Theorems 8 and 10 since the term $\Phi_{22}=-e^{-\alpha \tau_{M}}\left(K_{3}^{T}+K_{3}\right)-$ $e^{-\alpha \tau_{M}}\left(K_{4}^{T}+K_{4}\right)-e^{-\alpha \tau_{M}}\left(R_{2}^{T}+R_{2}\right)+e^{-\alpha \tau_{M}}\left(S^{T}+S\right)-(1-$ $\mu) e^{-\alpha \tau_{M}} M$ can be negative definite by choosing appropriate $K_{3}, K_{4}, R_{2}, S, M$ when $\mu>1$. Obviously, the results in this paper have more scope of application than the one in [11].

Remark 14. The reachable set of system (1) can be minimized by solving the following optimization problem for a scalar $\delta>$ 0 :

$$
\begin{array}{ll}
\min & \bar{\delta}\left(\bar{\delta}=\frac{1}{\delta}\right) \\
\text { s.t. } & \begin{cases}\text { (a) } & P \geq \delta I, \\
\text { (b) } & (34)-(35) \text { or } \quad(38)-(39)\end{cases}
\end{array}
$$

Remark 15. It is clear to see that radius $r$ is smallest if $\delta=$ $\min _{i=1,2, \ldots, N}$ is largest in inequalities (35) and (39). Therefore, one can consider $P_{i}(i=1,2, \ldots, N)$ as decision variable with the additional requirement (42).

Remark 16. It should be noted that the matrix inequalities in Theorems 7-10 cannot be simplified to LMIs. However, when $\alpha$ is fixed, then the matrix inequalities reduce to LMIs. 
Hence, we can use MATLAB's Toolbox to solve the matrix inequalities in Theorems 7-10.

Remark 17. The approach is likely to help further work in this area. It may be used to improve estimate partial state bounding for neural networks with time-varying delays, such as [3].

\section{Examples}

In this section, four numerical examples will be presented to show the validity of the main results derived in this paper.

Example 1. Consider the following uncertain time-delayed system with parameters:

$$
\begin{gathered}
A+A_{1}=\left[\begin{array}{cc}
-2 & 0 \\
0 & -0.7
\end{array}\right], \quad A+A 2=\left[\begin{array}{cc}
-2 & 0 \\
0 & -1.1
\end{array}\right], \\
D+D_{1}=\left[\begin{array}{cc}
-1 & 0 \\
-1 & -0.9
\end{array}\right], \quad D+D_{2}=\left[\begin{array}{cc}
-1 & 0 \\
-1 & -1.1
\end{array}\right], \\
B+B_{1}=\left[\begin{array}{c}
-0.5 \\
1
\end{array}\right]=B+B_{2}, \quad w^{T}(t) w(t) \leq 1 .
\end{gathered}
$$

By solving optimization problems (42), computed $\bar{\delta}$ 's for the case $\tau_{m} \leq \tau(t) \leq \tau_{M}$ with different values of $\tau_{M}$ are listed in Table 1. Computed $\bar{\delta}$ 's for different values of $\mu$ with $\tau_{M}=$ 0.7 and $\tau_{M}=0.75$ for the case $\tau_{m} \leq \tau(t) \leq \tau_{M}, \dot{\tau}(t) \leq \mu$ are obtained in Tables 2 and 3, respectively. It is clear to see that the proposed method in this paper yields tighter bounds than literatures $[6,8,11]$.

Example 2. Consider the following uncertain time-delayed system with parameters

$$
\begin{gathered}
A+A_{1}=\left[\begin{array}{cc}
0 & -0.0936 \\
1 & -0.685
\end{array}\right], \quad A+A 2=\left[\begin{array}{cc}
0 & -0.1464 \\
1 & -0.245
\end{array}\right], \\
D+D_{1}=\left[\begin{array}{cc}
-0.1 & -0.35 \\
0 & 0.3
\end{array}\right]=D+D_{2}, \\
B+B_{1}=\left[\begin{array}{c}
-1 \\
1
\end{array}\right]=B+B_{2}, \quad \mu=0, \tau_{m}=0, \tau_{M}=0.1, \\
w^{T}(t) w(t) \leq 1 .
\end{gathered}
$$

Computed $\bar{\delta}$ 's for the case $\tau_{m} \leq \tau(t) \leq \tau_{M}, \dot{\tau}(t) \leq \mu$ with $\tau_{m}=0, \tau_{M}=0.1$ are listed in Table 4 to compare with the ones in $[6,8,11]$. It should be noted that there is no feasible solution employing the approaches in $[6,11]$, and the derived method in this paper yields much tighter bounding than [8]. Hence, the proposed method leads to a wider application range.
TABLE 4: Computed $\bar{\delta}$ 's of Example 2 for the case $\tau_{m} \leq \tau(t) \leq \tau_{M}$, $\dot{\tau}(t) \leq \mu$ with $\tau_{m}=0, \tau_{M}=0.1$.

\begin{tabular}{lcccc}
\hline Method & {$[6]$} & {$[11]$} & {$[8]$} & Theorem 10 \\
\hline $\bar{\delta}$ & - & - & $2.8686 \times 10^{4}$ & 11.96 \\
\hline
\end{tabular}

TABLE 5: Computed radiuses r's of Example 3.

\begin{tabular}{lccccc}
\hline Method & {$[6]$} & {$[11]$} & {$[8]$} & {$[10]$} & Theorem 9 \\
\hline Case (1) & $\sqrt{19.71}$ & - & $\sqrt{5.3}$ & $\sqrt{1.2}$ & $\sqrt{1.17}$ \\
Case (2) & $\sqrt{19.71}$ & - & $\sqrt{5.3}$ & $\sqrt{1.1}$ & $\sqrt{0.87}$ \\
\hline
\end{tabular}

Example 3. Consider the following uncertain time-delayed system with parameters

$$
\begin{gathered}
A+A_{1}=\left[\begin{array}{cc}
-2 & 0 \\
0 & -0.7
\end{array}\right], \quad A+A 2=\left[\begin{array}{cc}
-2 & 0 \\
0 & -1.1
\end{array}\right], \\
D+D_{1}=\left[\begin{array}{cc}
-1 & 0 \\
-1 & -0.9
\end{array}\right], \quad D+D_{2}=\left[\begin{array}{cc}
-1 & 0 \\
-1 & -1.1
\end{array}\right], \\
B+B_{1}=\left[\begin{array}{c}
-0.5 \\
1
\end{array}\right]=B+B_{2}, \quad w^{T}(t) w(t) \leq 1,
\end{gathered}
$$

and time-varying delay $\tau(t)$ is considered in two cases:

(1) $\tau(t)$ is nondifferentiable, $0 \leq \tau(t) \leq 0.7$;

(2) $\tau(t)$ is nondifferentiable, $0.1 \leq \tau(t) \leq 0.7$.

From Theorem 9, computed radiuses $r$ 's for case (1) and case (2) are listed in Table 5. These results are compared to the ones in $[6,8,10,11]$. It is clear to see that our results decrease radiuses of the ellipsoid.

Example 4. Consider the following uncertain time-delayed system with parameters:

$$
\begin{aligned}
\dot{z}(t)= & {\left[\begin{array}{cc}
-2 & 0 \\
0 & -0.7
\end{array}\right] z(t)+\left[\begin{array}{cc}
-1 & 0 \\
-1 & -0.9
\end{array}\right] z(t-\tau(t)) } \\
& +\left[\begin{array}{c}
-0.5 \\
1
\end{array}\right] w(t),
\end{aligned}
$$

and $w^{T}(t) w(t) \leq 1$

By employing the method of Theorem 8 in this paper, $r$ 's for different values of $\tau(t)$ with $\mu=0$ are listed in Table 6 . It is easy to see that bounds obtained in this paper are better than the ones of literatures $[8-12,14]$.

\section{Conclusions}

In this paper, sufficient conditions for the existence of a ball that binds the reachable set of linear uncertain polytopic systems with disturbance and time-varying delays have been proposed in terms of Lyapunov-Krasovskii functional theory, delay decomposition technique, and reciprocally convex method. The proposed Lyapunov-Krasovskii functionals 
TABLE 6: Computed $r$ 's of Example 4 for different values of $\tau$ with $\mu=0$.

\begin{tabular}{|c|c|c|c|c|c|}
\hline$\tau$ & 0.1 & 0.3 & 0.5 & 0.7 & 0.9 \\
\hline [11] & $\sqrt{0.83}$ & $\sqrt{1.28}$ & $\sqrt{1.94}$ & $\sqrt{2.90}$ & $\sqrt{4.46}$ \\
\hline [10] & $\sqrt{0.74}$ & $\sqrt{0.92}$ & $\sqrt{1.36}$ & $\sqrt{2.30}$ & $\sqrt{3.51}$ \\
\hline [12] & $\sqrt{0.68}$ & $\sqrt{0.80}$ & $\sqrt{0.97}$ & $\sqrt{1.64}$ & $\sqrt{3.22}$ \\
\hline [8] & $\sqrt{0.66}$ & $\sqrt{0.75}$ & $\sqrt{0.94}$ & $\sqrt{1.61}$ & $\sqrt{3.14}$ \\
\hline [9] & $\sqrt{0.66}$ & $\sqrt{0.75}$ & $\sqrt{0.94}$ & $\sqrt{1.61}$ & $\sqrt{3.14}$ \\
\hline [14] & $\sqrt{0.66}$ & $\sqrt{0.75}$ & $\sqrt{0.94}$ & $\sqrt{1.61}$ & $\sqrt{3.14}$ \\
\hline Theorem 8 & $\sqrt{0.41}$ & $\sqrt{0.71}$ & $\sqrt{0.92}$ & $\sqrt{1.25}$ & $\sqrt{2.74}$ \\
\hline
\end{tabular}

contain triple integral terms, which lead to tighter bounding than previous literatures. Numerical examples have been given to illustrate the effectiveness and improvement of the proposed methods. These results are likely to help further work in this area. One future work is to extend the results in this technical note to linear neutral systems and linear mixed delay systems.

\section{Conflict of Interests}

The author declares that there is no conflict of interests regarding the publication of this paper.

\section{Acknowledgments}

This work was supported by National Natural Science Foundation of China under Grant no. 61273015, the Fundamental Research Funds for the Central Universities under Grants nos. 12NZYQN17, 12NZYQN21, and 13NZYBS07, and the State Ethnic Affairs Commission Project under Grant no. $12 X N Z 2003$.

\section{References}

[1] J. K. Tian, S. M. Zhong, and Y. Wang, "Improved exponential stability criteria for neural networks with time-varying delays," Neurocomputing, vol. 97, no. 11, pp. 164-173, 2012.

[2] W. Lee II, S. Y. Lee, and P. Park, "Improved criteria on robust stability and $H_{\infty}$ performance for linear systems with interval time-varying delays via new triple integral functionals," Applied Mathematics and Computation, vol. 243, pp. 570-577, 2014.

[3] J. Cheng, S. Zhong, Q. Zhong, H. Zhu, and Y. Du, "Finite-time boundedness of state estimation for neural networks with timevarying delays," Neurocomputing, vol. 129, pp. 257-264, 2014.

[4] K. Q. Gu, "An integral inequality in the stability problem of time-delay systems," in Proceedings of the 39th IEEE Confernce on Decision and Control, pp. 2805-2810, Sydney, Australia, December 2000.

[5] J. Sun, G. P. Liu, J. Chen, and D. Rees, "Improved delay-rangedependent stability criteria for linear systems with time-varying delays," Automatica, vol. 46, no. 2, pp. 466-470, 2010.

[6] E. Fridman and U. Shaked, "On reachable sets for linear systems with delay and bounded peak inputs," Automatica, vol. 39, no. 11, pp. 2005-2010, 2003.

[7] Z. Zuo, Y. Chen, Y. Wang, D. W. Ho, M. Z. Chen, and H. Li, "A note on reachable set bounding for delayed systems with polytopic uncertainties," Journal of the Franklin Institute, vol. 350, no. 7, pp. 1827-1835, 2013.

[8] Z. Q. Zuo, D. W. C. Ho, and Y. J. Wang, "Reachable set bounding for delayed systems with polytopic uncertainties: the maximal Lyapunov-Krasovskii functional approach," Automatica, vol. 46, no. 5, pp. 949-952, 2010.

[9] C. C. Shen and S. M. Zhong, "The ellipsoidal bound of reachable sets for linear neutral systems with disturbances," Journal of the Franklin Institute. Engineering and Applied Mathematics, vol. 348, no. 9, pp. 2570-2585, 2011.

[10] P. T. Nam and P. N. Pathirana, "Further result on reachable set bounding for linear uncertain polytopic systems with interval time-varying delays," Automatica, vol. 47, no. 8, pp. 1838-1841, 2011.

[11] J.-H. Kim, "Improved ellipsoidal bound of reachable sets for time-delayed linear systems with disturbances," Automatica, vol. 44, no. 11, pp. 2940-2943, 2008.

[12] O. M. Kwon, S. M. Lee, and J. H. Park, "On the reachable set bounding of uncertain dynamic systems with time-varying delays and disturbances," Information Sciences, vol. 181, no. 17, pp. 3735-3748, 2011.

[13] S. Boyd, L. El Ghaoui, E. Feron, and V. Balakrishnan, Linear Matrix Inequalities in Systems and Control Theory, SIAM, Philadelphia, Pa, USA, 1994.

[14] Z. Zuo, D. W. Ho, and Y. Wang, "Reachable set estimation for linear systems in the presence of both discrete and distributed delays," IET Control Theory \& Applications, vol. 5, no. 15, pp. 1808-1812, 2011.

[15] Z. Q. Zuo, Z. Q. Wang, Y. P. Chen, and Y. J. Wang, "A nonellipsoidal reachable set estimation for uncertain neural networks with time-varying delay," Communications in Nonlinear Science and Numerical Simulation, vol. 19, no. 4, pp. 1097-1106, 2014.

[16] Z. Q. Zuo, Y. P. Chen, and Y. J. Wang, "New criteria of reachable set estimation for time delay systems subject to polytopic uncertainties," in Proceedings of the 7th IFAC Symposium on Robust Control Design (ROCOND '12), pp. 231-235, Aalborg, Denmark, June 2012.

[17] N. D. That, P. T. Nam, and Q. P. Ha, "Reachable set bounding for linear discrete-time systems with delays and bounded disturbances," Journal of Optimization Theory and Applications, vol. 157, no. 1, pp. 96-107, 2013.

[18] L. V. Hien and H. M. Trinh, "A new approach to state bounding for linear time-varying systems with delay and bounded disturbances," Automatica, vol. 50, no. 6, pp. 1735-1738, 2014.

[19] W. J. Rugh, Linear System Theory, Prentice Hall Press, Englewood Cliffs, NJ, USA, 2nd edition, 1996. 


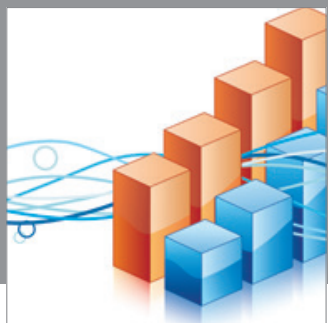

Advances in

Operations Research

mansans

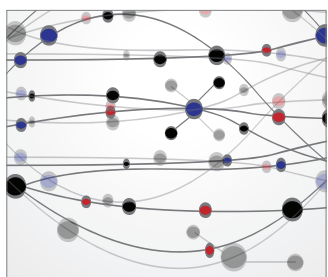

The Scientific World Journal
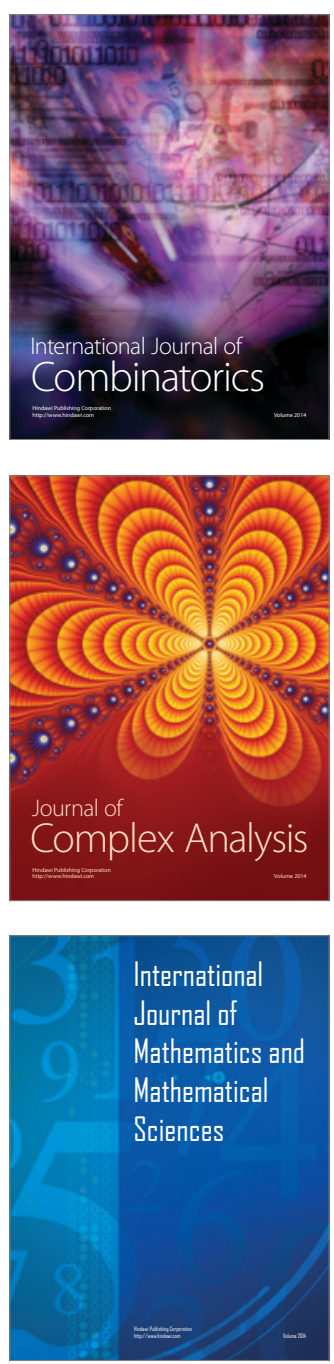
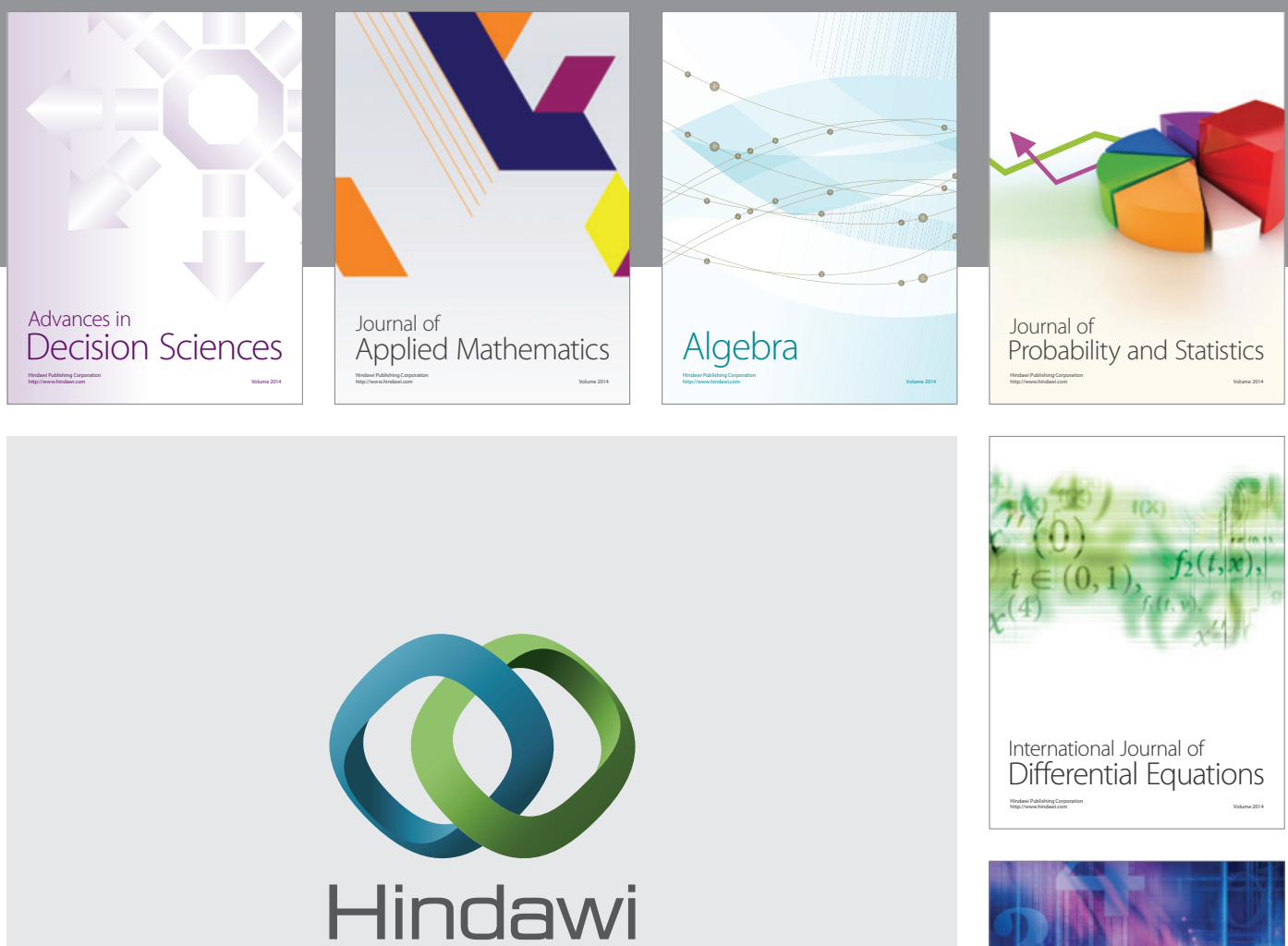

Submit your manuscripts at http://www.hindawi.com
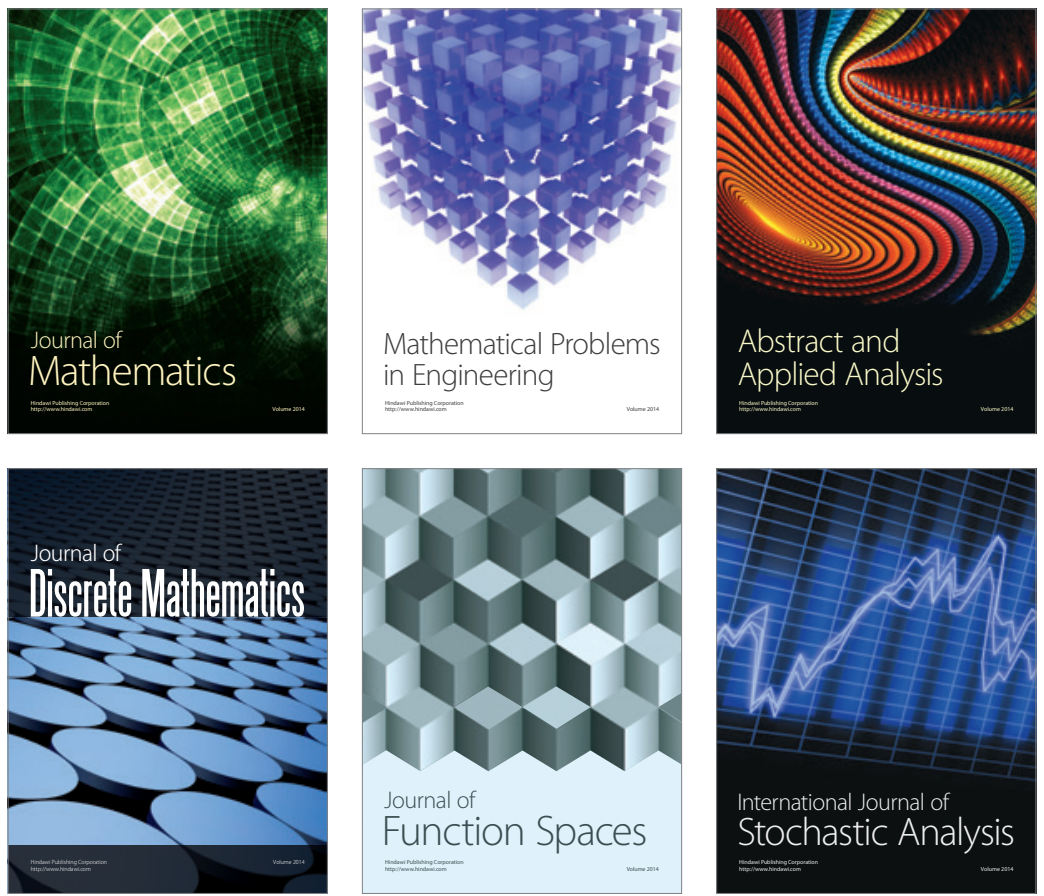

Journal of

Function Spaces

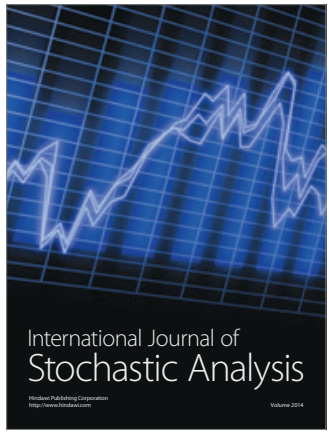

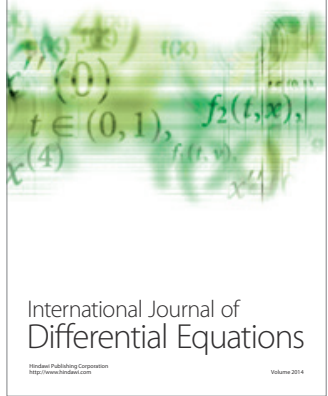
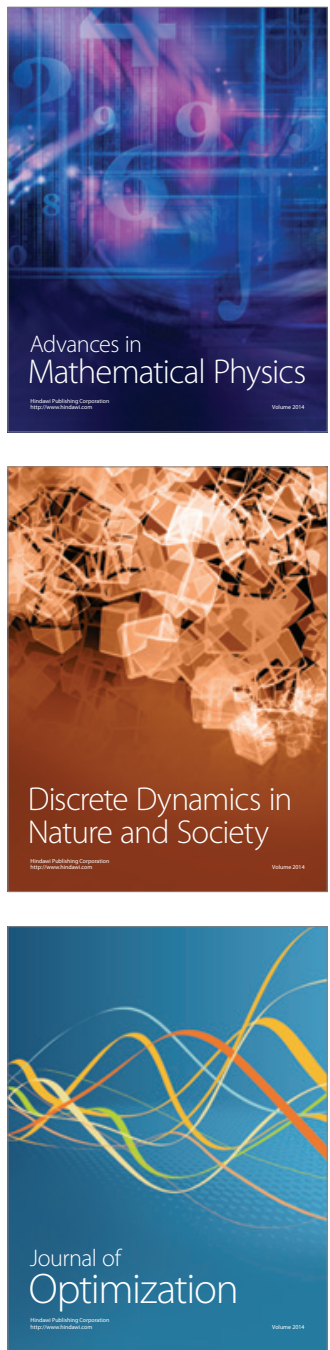